\title{
Capicua suppresses colorectal cancer progression via repression of ETV4 expression
}

Jeon-Soo Lee ${ }^{1 \dagger}$, Eunjeong Kim ${ }^{1 \dagger}$, Jongeun Lee ${ }^{1 \dagger}$, Donghyo Kim', Hyeongjoo Kim³ ${ }^{3}$ Chang-Jin Kim Sanguk Kim ${ }^{1,2}$, Dongjun Jeong ${ }^{3,4^{*}}$ and Yoontae Lee Le, $^{1,5^{*}}$ (D)

\begin{abstract}
Background: Although major driver gene mutations have been identified, the complex molecular heterogeneity of colorectal cancer (CRC) remains unclear. Capicua (CIC) functions as a tumor suppressor in various types of cancers; however, its role in CRC progression has not been examined.
\end{abstract}

Methods: Databases for gene expression profile in CRC patient samples were used to evaluate the association of the levels of CIC and Polyoma enhancer activator 3 (PEA3) group genes (ETS translocation variant 1 (ETV1), ETV4, and ETV5), the best-characterized CIC targets in terms of CIC functions, with clinicopathological features of CRC. CIC and ETV4 protein levels were also examined in CRC patient tissue samples. Gain- and loss-of function experiments in cell lines and mouse xenograft models were performed to investigate regulatory functions of CIC and ETV4 in CRC cell growth and invasion. qRT-PCR and western blot analyses were performed to verify the CIC regulation of ETV4 expression in CRC cells. Rescue experiments were conducted using siRNA against ETV4 and CIC-deficient CRC cell lines.

Results: CIC expression was decreased in the tissue samples of CRC patients. Cell invasion, migration, and proliferation were enhanced in CIC-deficient CRC cells and suppressed in $\mathrm{CIC}$-overexpressing cells. Among PEA3 group genes, ETV4 levels were most dramatically upregulated and inversely correlated with the CIC levels in CRC patient samples. Furthermore, derepression of ETV4 was more prominent in CIC-deficient CRC cells, when compared with that observed for ETV1 and ETV5. The enhanced cell proliferative and invasive capabilities in CIC-deficient CRC cells were completely recovered by knockdown of ETV4.

Conclusion: Collectively, the CIC-ETV4 axis is not only a key module that controls CRC progression but also a novel therapeutic and/or diagnostic target for CRC.

Keywords: Colorectal cancer, Capicua (CIC), Polyoma enhancer activator 3 (PEA3), ETS translocation variant 4 (ETV4)

*Correspondence: juny1024@sch.ac.kr; yoontael@postech.ac.kr ${ }^{\dagger}$ Jeon-Soo Lee, Eunjeong Kim and Jongeun Lee contributed equally to this work

${ }^{4}$ Department of Pathology, College of Medicine, Soonchunhyang University, Room 601, 31 Soonchunhyang 6gil, Dongnam-gu, Cheonan, Chungnam 31151, Republic of Korea

5 POSTECH Biotech Center, Room 388, 77 Cheongam-Ro, Nam-Gu, Pohang, Gyeongbuk 37673, Republic of Korea

Full list of author information is available at the end of the article

\section{Background}

Of the 36 various types of cancers diagnosed worldwide, colorectal cancer (CRC) ranks third among the most frequently occurring cancers and second in terms of cancer mortalities [1]. Common genetic alterations responsible for the development and progression of CRC include inactivation of the tumor suppressors Adenomatosis polyposis coli $(A P C)(\sim 70 \%)$ and TP53 ( 60\%) and mutational activation of KRAS ( 40\%) [2-7]. For the treatment of CRC, targeted therapy drugs such as bevacizumab and cetuximab, which are inhibitors of

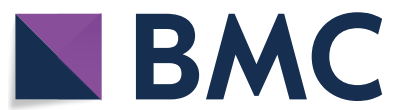

(c) The Author(s) 2020. This article is licensed under a Creative Commons Attribution 4.0 International License, which permits use, sharing, adaptation, distribution and reproduction in any medium or format, as long as you give appropriate credit to the original author(s) and the source, provide a link to the Creative Commons licence, and indicate if changes were made. The images or other third party material in this article are included in the article's Creative Commons licence, unless indicated otherwise in a credit line to the material. If material is not included in the article's Creative Commons licence and your intended use is not permitted by statutory regulation or exceeds the permitted use, you will need to obtain permission directly from the copyright holder. To view a copy of this licence, visit http://creativeco mmons.org/licenses/by/4.0/. The Creative Commons Public Domain Dedication waiver (http://creativecommons.org/publicdomain/ zero/1.0/) applies to the data made available in this article, unless otherwise stated in a credit line to the data. 
angiogenesis and the epidermal growth factor receptor (EGFR) pathway, respectively, have been actively developed [8]. However, these inhibitors cannot be used for the effective treatment of all CRC patients. Therefore, additional therapeutic strategies for the treatment of CRC must be developed.

Capicua (CIC) is a transcriptional repressor containing a high mobility group (HMG) box domain and a C-terminal motif that are evolutionarily conserved from Caenorhabditis elegans to humans [9-14]. Through the HMG box and C-terminal domains, CIC recognizes specific octameric DNA sequences $\left(5^{\prime}-\mathrm{T}(\mathrm{G} / \mathrm{C}) \mathrm{AATG}(\mathrm{A} / \mathrm{G})\right.$ $\left.(\mathrm{A} / \mathrm{G})-3^{\prime}\right)$ to regulate the expression of its target genes $[12,15,16]$. There are two main isoforms of CIC, the short (CIC-S) and long (CIC-L) form, which are distinguished by their amino-terminal regions $[17,18]$. It is known that CIC is regulated by extracellular signal-regulated kinase (ERK), which is a downstream kinase of the RAS/RAF/MEK signaling cascade. Activation of the MAPK pathway (RAS/RAF/MEK/ERK) results in phosphorylation of $\mathrm{CIC}$, and this ultimately leads to degradation or cytoplasmic localization of CIC [19-21]. CIC controls several essential processes including cell proliferation and tissue patterning in Drosophila [13, 22, 23]. In mammals, CIC is required for lung alveolarization, liver homeostasis, brain development and function, and immune cell homeostasis [24-28].

Accumulating evidence indicates that $\mathrm{CIC}$ functions as a tumor suppressor in various types of cancers. Previous studies have identified numerous $C I C$ mutations in patients suffering from various types of cancers, including soft tissue, brain, lung, gastric, prostate, and breast cancers [9, 29-32]. Additionally, chromosomal translocations that generate the CIC-DUX4 chimeric form have been identified in Ewing-like sarcomas [9, 33-35]. Either mutations in or loss of CIC can promote cancer progression via upregulating the expression of $P E A 3$ group genes (ETV1/ER81, ETV4/PEA3, and ETV5/ERM), the best characterized and reliable CIC target genes $[9,32$, $36,37]$. The PEA3 group factors are known as an oncogenic transcription factor, because the overexpression of these transcription factors promotes cancer cell proliferation and metastasis via activating the transcription of a subset of genes related to control of cell division and migration, such as matrix metalloprotease (MMP), vascular endothelial growth factor (VEGF), and telomerase reverse transcriptase (TERT) [38]. Several CIC mutations were found in the CRC patient samples ( 6 out of 74 samples) [39], and it is therefore conceivable that CIC may also be involved in the regulation of CRC progression. Regardless, the exact role of CIC in the suppression of CRC progression and the CIC target genes involved in this process remain to be investigated.
In this study, we examined the association of CIC and PEA3 group transcription factors with CRC clinicopathology by conducting analyses of the TCGA dataset and tissue samples derived from CRC patients. We also investigated the molecular basis underlying CIC-mediated regulation of CRC progression through the use of CRC cell lines and mouse xenograft models. Our study identifies the CIC-ETV4 axis as a key molecular module that controls CRC progression.

\section{Materials and methods \\ Cell culture}

HCT116 (ATCC_CCL-247 ${ }^{\mathrm{TM}}$ ) and HT29 (ATCC_HTB$38^{\mathrm{TM}}$ ) colorectal cancer cells were cultured in DMEM (Welgene, Gyeongsan, Republic of Korea) containing 10\% FBS (Welgene, Gyeongsan, Republic of Korea) and 1\% penicillin/streptomycin (Gibco, MA, USA). Cells were incubated at $37^{\circ} \mathrm{C}$ in a $5 \% \mathrm{CO}_{2}$ incubator.

\section{Mice}

Male BALB/C nude mice (5-week-old) were purchased from OrientBio (Seongnam, Republic of Korea) and were subjected to acclimatization for 1 week. They were then used for the in vivo tumor formation assay. Mice were fed standard rodent chow and water ad libitum and maintained in a specific pathogen-free animal facility under standard $12 \mathrm{~h}$ light $/ 12 \mathrm{~h}$ dark cycle. All experimental procedures of animal studies followed the guidelines and regulations approved by the POSTECH Institutional Animal Care and Use Committee (IACUC).

\section{Human tissue samples}

Human tissue samples were obtained from Soonchunhyang University Hospital (Cheonan, Republic of Korea). The colon tissue samples from 13 patients with CRC were used in this study. Informed consent was obtained from all patients. All procedures were approved by the Soonchunhyang University Hospital Institutional Review Board (SCHCA 2018-07-061-003).

\section{Generation of viruses and stable cell lines}

ETV4 shRNA and CIC sgRNA cassettes were cloned into MSCV-LTRmiR30-PIG (LMP) and lentiCRISPR v2 plasmids, respectively, according to the manufacturer's manuals. HCT116 and HT29 CRC cells were infected with viral supernatants in the presence of polybrene (SigmaAldrich, MO, USA). After 24-48 h, the cells were selected using $2 \mu \mathrm{g} / \mathrm{ml}$ of puromycin (Gibco, MA, USA) for $48 \mathrm{~h}$. For overexpression of CIC-S and ETV4, the cloned pHAGE-FLAG-CIC-S, pHAGE-ETV4, and pHAGE control plasmids were used. The lentivirus production process was described previously [36]. Viral supernatants were collected at $48 \mathrm{~h}$ post-transfection and were used to 
infect the HCT116 or HT29 cells for 3 sequential days. The cells were used for further biochemical assays as specified in each experiment.

\section{siRNA transfection}

ETV4 siRNA (siETV4) was purchased from Bioneer (Daejun, Republic of Korea). The sequences are as follows: siETV4 sense; 5'- GAGGAAUUCAGCUCAGCU UdTdT $-3^{\prime}$, and antisense; $5^{\prime}$ - AAGCUGAGCUGAAUU CCUCdTdT $-3^{\prime}$. One day prior to transfection, $1 \times 10^{5}$ cells were plated in $60 \mathrm{~mm}$ plates. After $24 \mathrm{~h}$, the cells were transfected with $120 \mathrm{pmol}$ of siRNA duplexes using Dharmafect 1 , according to the manufacturer's instructions. After $72 \mathrm{~h}$, the cells were used for further biochemical assays as specified in each experiment.

\section{qRT-PCR}

Total RNA was extracted using RiboEX (GeneAll, Seoul, Republic of Korea). cDNA was synthesized using a GoScript $^{\mathrm{TM}}$ Reverse Transcript kit (Promega, WI, USA), according to the manufacturer's instructions. SYBR Green PCR Mixture (Toyobo, NY, USA) was used for qRT-PCR analysis. Expression data were acquired using a StepOnePlus ${ }^{\text {TM }}$ Real-Time PCR System (Applied Biosystems, CA, USA). Expression levels of each target were calculated using the $2^{-\Delta \Delta C t}$ method and were presented as relative mRNA expression. The sequences of primers used for qRT-PCR were previously described [37].

\section{Cell lysis and immunoblotting}

Cells were harvested and lysed in RIPA buffer $(50 \mathrm{mM}$ Tris (pH 7.4), $150 \mathrm{mM} \mathrm{NaCl}, 0.5 \%$ sodium deoxycholate, $0.1 \%$ SDS, and $1 \%$ Triton X-100) containing complete protease inhibitor cocktail tablets (Roche, Basel, Switzerland) by sonication. The lysates of CRC patients' tissue samples were also prepared by sonication in RIPA buffer. The concentration of cell proteins was determined via the BCA assay. Western blot analysis was performed as described previously [25]. Generation of rabbit polyclonal anti-CIC antibody was previously described [25]. Anti-ETV4 (10684-1-AP) antibody was purchased from Proteintech (IL, USA). Anti- $\beta$-ACTIN (sc-47778) antibody was purchased from Santa-Cruz Biotechnology (TX, USA). HRP-conjugated secondary antibody was purchased from Pierce Thermo Scientific (MA, USA). The western blot images were obtained using ImageQuant LAS 500 (GE Healthcare Life Science, PA, USA).

\section{Cell growth assay}

Stably infected cells $\left(7 \times 10^{3}\right.$ cells $)$ were seeded into each well of 24-well plates. The cells were trypsinized and stained with Trypan Blue (Sigma-Aldrich, MO, USA). The number of viable cells was counted using a hemocytometer every day for 4 days. For cell growth assays of siRNA-treated CIC knockout HCT116 or HT29 cells, $7 \times 10^{3}$ cells were seeded into 24 -well plates 1 day before transfection, and then siRNAs were transfected using Dharmafect 1 (Dharmacon, CO, USA) and set as day " 0 ". The cells were trypsinized and stained with Trypan Blue. The number of viable cells was counted using a hemocytometer every day for 4 days.

\section{In vitro migration and invasion assay}

A 24-well trans-well plate (8- $\mu \mathrm{m}$ pore size, SPL, Pocheon, Republic of Korea) was used to measure the migratory and invasive abilities of each cell line. For trans-well migration assays, $5 \times 10^{4}$ cells were plated in the top chamber lined with a non-coated membrane. The inserts were cultured in a well of 10\% FBS containing media and were incubated for $6 \mathrm{~h}$. They were then removed, washed with PBS, stained with formalin $/ 0.1 \%$ crystal violet solution, and analyzed under a ZEISS Axioplan2 microscope. Multiple 5-10 images per insert were acquired, and the average counts were calculated. For invasion assays, chamber inserts were coated with $16 \mu \mathrm{l} / \mathrm{ml}$ of Matrigel (BD Biosciences, MA, USA) with DMEM/F12 media (Gibco, MA, USA) and dried overnight under sterile conditions. Next, $1 \times 10^{5}$ cells were plated in the top chamber. The inserts were cultured in a well of $10 \%$ FBScontaining media and incubated for $48 \mathrm{~h}$. The same staining method used in the migration assay was applied.

\section{In vivo tumor growth assay}

For xenograft tumor growth assays, control and CIC KO cells $\left(5 \times 10^{6}\right.$ cells) were subcutaneously injected into the posterior flank of 6 -week-old male BALB/C nude mice. Seven days after inoculation, the tumor size was measured every week for 12-13 weeks. The tumor volume was calculated as $1 / 2 \times$ (largest diameter) $\times$ (smallest diameter) $)^{2}$.

\section{Tissue microarray and immunohistochemistry}

The colorectal cancer tissue microarray (CO2085b) was purchased from Biomax (MD, USA). Formalin-fixed paraffin-embedded specimens were deparaffinized and stained with rabbit polyclonal anti-ETV4 antibody (1:500 dilution). Each sample stained with anti-ETV4 antibody was scored as negative $(-)$, weak $(+)$, or strong $(++)$ according to the staining intensity. These scores were determined independently by two pathologists in a blinded manner. Tissue samples from 9 CRC patients were provided by Soonchumhyang University Hospital (Cheonan, Republic of Korea). Formalin-fixed paraffin-embedded specimens were deparaffinized, and the antigens were retrieved via a citrate-buffered $(\mathrm{pH}$ 6.0) solution method. After blocking endogenous peroxidase 
activity, immunohistochemistry of CIC and ETV4 was performed using a VECTASTAIN Elite ABC HRP Kit (Vector Labs, CA, USA) according to the manufacturer's instruction. Specimens were stained with home-made rabbit polyclonal anti-CIC antibody (1:500 dilution) [25] or anti-ETV4 antibody (1:500 dilution). The color reaction was performed using a DAB kit (Vector Labs, CA, USA). Then, the sections were counterstained with Mayer's hematoxylin, dehydrated, and mounted. Images were acquired under an OLYMPUS BX41 microscope and analyzed by SPOT Basic image capture software.

\section{TCGA database analysis}

Gene expression data from colorectal cancer and normal cells (mRNA, normalized RNAseq FPKM-UQ, July 2014) were retrieved from the TCGA database (provisional) using cBioPortal for cancer genomics during the diagnosed period from 1998 to 2013. Gene expression data were available for 453 CRC patients. Expression levels were $\log 2$ transformed. Clinical data including the tumor stage were downloaded from the TCGA portal in July 2014. Tumor stages were defined using the latest version of the American Joint Committee on cancer code at the time of diagnosis. Major tumor stages (I, II, III, or IV) were investigated for differences in gene expression. Expression levels of $C I C$ and PEA3 group genes after normalization were compared among the tumor stages. $P$ values were calculated using a Mann-Whitney U test comparing the expression values in the patient samples at each tumor stages. The detailed clinical and pathological characteristics of CRC patients in TCGA database are listed in Additional file 1: Table S2.

\section{Statistical analysis}

For statistical analysis, all experiments were performed more than thrice independently. Data are presented as mean \pm standard error. The quantitative data were compared between groups using the Student's $t$ test (twotailed, two-sample unequal variance). A value of $P<0.05$ was considered to be statistically significant.

\section{Results}

\section{Association of CIC with CRC}

To gain insight into the association between CIC and CRC, we searched for CIC mutations and expression changes in the CRC patient samples using public cancer databases. Several mutations in the CIC gene have been identified from CRC patient samples according to the information from the cBioPortal database [40] (Additional file 2: Fig. S1a). Moreover, CRC ranked fourth among the 27 different types of cancers in terms of the number of CIC mutations identified in each tumor type (Additional file 1: Table S1). Analyses of the datasets for
CRC patients from The Cancer Genome Atlas (TCGA) and Catalogue of Somatic Mutations in Cancer (COSMIC) databases revealed that CIC levels were slightly decreased in the colorectal tumor samples compared with the normal tissues (Fig. 1a and b, Additional file 1: Table S2, and Additional file 2: Fig. S1b). Considering that $\mathrm{CIC}$ expression is robustly regulated by the RAS/ MAPK signaling pathway at posttranslational levels [12, $32,37]$, we examined the CIC protein levels in CRC tissue samples. Notably, the CIC expression was dramatically decreased in the CRC regions compared with that in the normal tissue area (Fig. 1c, Additional file 1: Table S3, and Additional file 3: Fig. S2). This result was confirmed by western blot analysis (Fig. 1d and Additional file 1: Table S4). Collectively, these data suggest that loss of CIC function is potentially associated with progression of CRC.

\section{CIC suppresses CRC cell growth, invasion, and migration} To determine that $\mathrm{CIC}$ regulates $\mathrm{CRC}$ progression, we examined the cell proliferative, invasive, and migratory capabilities in CRC cells by varying the expression of $\mathrm{CIC}$. We prepared CIC-deficient (CIC $\mathrm{KO}$ ) cells in two different CRC cell lines (HCT116 and HT29) using CIC-targeted (exon4 of CIC gene) CRISPR-Cas9 system. Moreover, we generated CRC cells that overexpress CIC-S by a serial infection of lentivirus expressing CIC-S. Loss or overexpression of CIC expression was confirmed via immunoblotting (Fig. 2a). CIC deficiency promoted cell proliferation in both CRC cell lines, whereas the forced expression of CIC-S suppressed it (Fig. 2b). We confirmed these results in vivo using xenograft mouse models. We subcutaneously transplanted the control and $\mathrm{CIC} \mathrm{KO} \mathrm{HCT116} \mathrm{cells} \mathrm{into} \mathrm{left} \mathrm{and} \mathrm{right} \mathrm{posterior}$ flank of the same BALB/c athymic mice, respectively, and measured the tumor volumes every week. The CIC KO cells grew more rapidly and formed larger tumor mass than the control cells (Fig. 2c). Furthermore, we tested the invasive and migratory capacities of CIC-deficient or CIC-overexpressing CRC cells. The invasive and migratory capabilities were negatively regulated by $\mathrm{CIC}$ in the CRC cells: there were more invaded and migrated cells in CIC KO CRC cells, whereas less in CIC-overexpressing cells (Figs. 2d, e). Collectively, these findings indicate that $\mathrm{CIC}$ suppresses CRC progression.

\section{ETV4 is most relevant to CRC progression among PEA3 group transcription factors}

Considering that $P E A 3$ group genes are known as oncogenic transcription factors [38] as well as direct target genes of CIC [9, 24, 31, 41-43], we examined the relevant factors for CRC progression among these three genes. The TCGA dataset analysis revealed that 

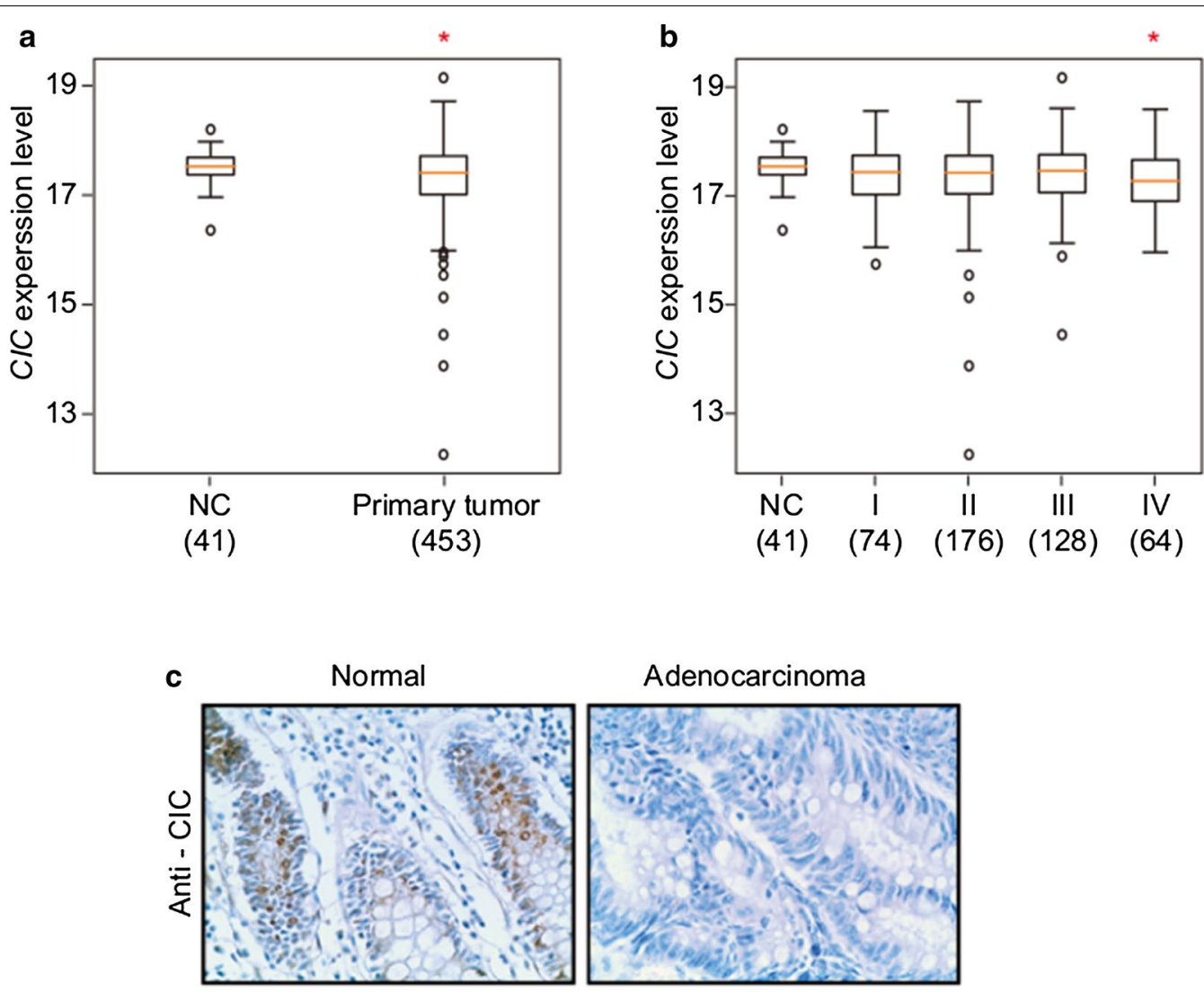

\section{Adenocarcinoma}

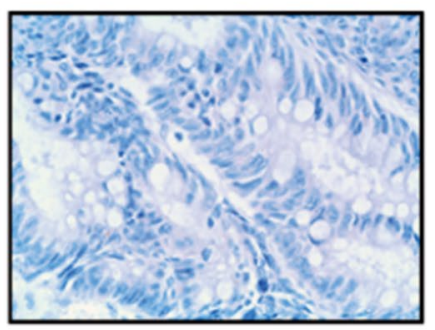

d
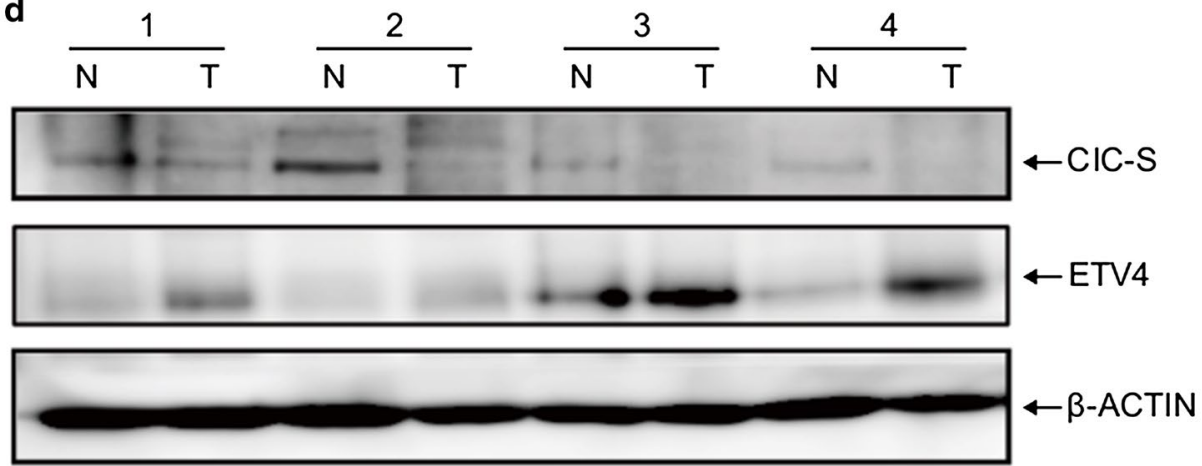

Fig. 1 Loss of CIC in colorectal cancer. a Analysis of the TCGA dataset for CIC mRNA levels in normal colon (NC) and primary colorectal tumor samples. The numbers in parentheses indicate the number of subjects in each group. ${ }^{*} P<0.05$. b Analysis of the TCGA dataset for CIC mRNA levels in normal colon (NC) and CRC samples of four different clinicopathological stages (I, II, III, and IV). The numbers in parentheses indicate the number of subjects in each group. ${ }^{*} P<0.05$. $\mathbf{c}$ Analysis of CIC protein levels in normal colon and CRC tissues by immunohistochemistry. $\mathbf{d}$ Western blot analysis for $\mathrm{CIC}$ and ETV4 levels in normal colon (N) and CRC (T) samples from the same patient with CRC. Samples from four CRC patients were subjected to this experiment

ETV4 and ETV5 were significantly upregulated across all stages of CRC (Fig. 3a), and that ETV4 expression was notably increased in the CRC samples compared with that in the normal colon tissues among the PEA3 group genes (Fig. 3a), consistent with previous findings that overexpression of ETV4 was frequently observed in samples from patients with CRC $[39,44,45]$. We confirmed the overexpression of ETV4 proteins in CRC tissue samples via immunohistochemistry (CRC tissue microarray and $10 \mathrm{CRC}$ patients' samples; Fig. 3b, Additional file 1: Table S3, and Additional file 4: Fig. S3) 
a

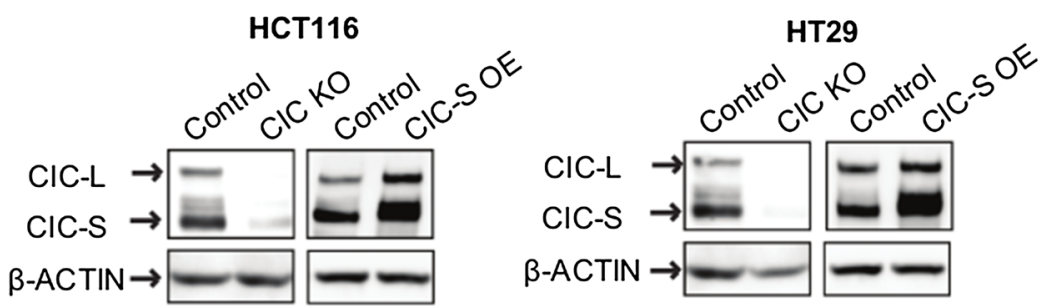

b

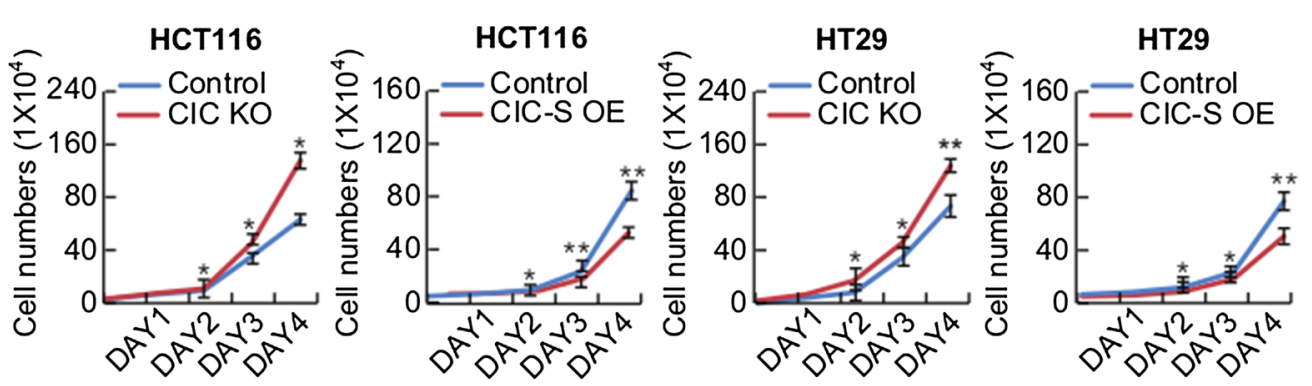

C
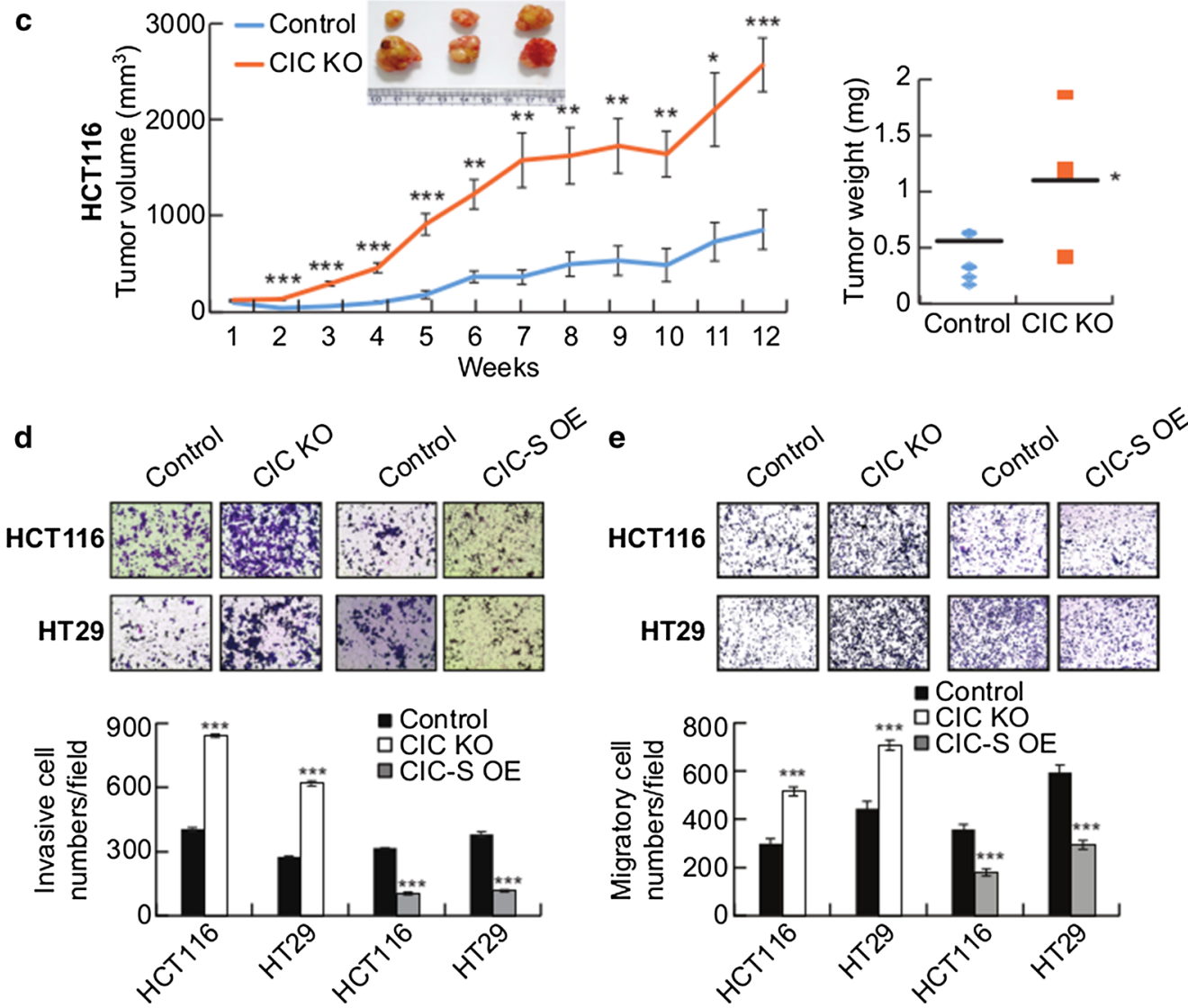

Fig. 2 CIC suppresses CRC progression. a Western blot analysis presenting the overexpression and knockout of CIC in HCT116 and HT29 CRC cells. b Cell growth assay of the CIC-S-overexpressing and CIC-deficient (CIC KO) CRC cells. c In vivo subcutaneous tumor growth curves of control and CIC KO HCT1 16 cells. $n=7$ per each group. The inset image is a representative image of xenograft tumors dissected from the mice after the last measurement of tumor size. The right panel is a graph for average weights of the dissected tumors. $\mathbf{d}$, e Matrigel invasion (d) and trans-well migration (e) assay of control, CIC KO, and CIC-overexpressing CRC cells. The bottom panels are bar graphs for quantification of cell invasiveness (d) and cell migration (e), respectively. Three independent experiments were performed. All error bars indicate s.e.m. ${ }^{*} P<0.05,{ }^{* *} P<0.01$, and ${ }^{* * *} P<0.001$ 


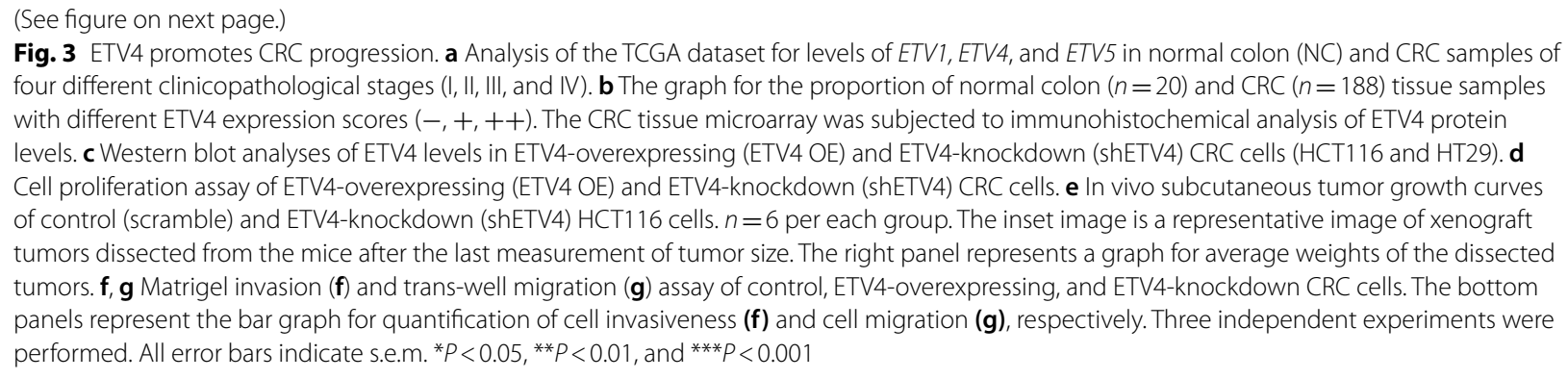

as well as western blot analysis (4 CRC patients' samples; Fig. 1d, Additional file 1: Table S4).

To verify that ETV4 has a tumor promoting activity in the CRC cells, we generated ETV4-overexpressing and ETV4-knockdown CRC cell lines (Fig. 3c). Overexpression of ETV4 promoted proliferation of CRC cells, whereas knockdown of ETV4 suppressed it (Fig. 3d). This result was also confirmed in xenograft mouse models (Fig. 3e). Moreover, increased and decreased invasive and migratory capabilities were observed in ETV4-overexpressing and ETV4-knockdown CRC cell lines, respectively (Fig. 3f, g). Collectively, these results suggest that ETV4 might be most critically associated with CRC progression among the PEA3 group transcription factors.

\section{CIC deficiency promotes CRC progression via ETV4 derepression}

To determine the transcription factor-target gene relationship between CIC and PEA3 group genes in CRC cells, we investigated alterations in expression of $P E A 3$ group genes by loss of CIC in CRC cells. Among the three genes, ETV4 and ETV5 were derepressed, and ETV4 had the highest fold increase in both CIC KO HCT116 and HT29 cells (Fig. 4a), suggesting that the regulation of ETV4 expression might be most considerably dependent on CIC in CRC cells compared with those of ETV1 and ETV5. The derepression of ETV4 was also confirmed at protein levels in CIC KO CRC cells (Fig. 4b). Consistent with these results, we have also observed that there was an inverse correlation between CIC and ETV4 expression in normal colon $(\mathrm{N})$ and $\mathrm{CRC}(\mathrm{T})$ samples from the same patient with CRC (Fig. 1d).

We finally determined whether the increased cell proliferation, invasion, and migration in the CIC-deficient CRC cells were due to the derepression of ETV4. To this end, we transfected the control and CIC KO CRC cells with either control siRNA or siRNA against ETV4 (siETV4). Treatment with siETV4 relieved the upregulation of ETV4 levels in CIC KO CRC cells (Fig. 4c). We then examined cell proliferation, invasion, and migration in this set of CRC cells. Knockdown of ETV4 substantially blocked the CIC deficiency-mediated promotion of cell proliferation (Fig. 4d), invasion (Fig. 4e), and migration (Fig. 4f), demonstrating that the CIC-ETV4 axis regulates CRC progression.

\section{Discussion}

Previous studies have shown that CIC functions as a tumor suppressor in various types of cancers, such as brain, lung, gastric, prostate, and liver cancers [30-32, 36, 37]. In most cases, CIC deficiency promotes cancer progression via derepression of $P E A 3$ group genes, and the degree of derepression of each member of the $P E A 3$ group genes is variable among cancer types: ETV 5 is the most significantly and dramatically upregulated in CICdeficient prostate cancer cells [36], while ETV4 is upregulated in liver cancer cells [37]. Our findings demonstrate that CIC functions as a tumor suppressor in CRC cells and highlight ETV4, among the PEA3 group transcription factors, as a strong promoter of cancer progression and as a critical target of CIC in the context of CRC.

Analyses of the TCGA dataset and tissue samples from CRC patients revealed that CIC expression was more prominently reduced in CRC patients at the protein level than it was at the mRNA level. Somatic mutations of KRAS occur in over $40 \%$ of sporadic CRC, and abnormal activation of mutated KRAS affects the activation of the downstream molecules [46, 47]. As activation of the RAS/MAPK signaling pathway suppresses CIC activity via degradation or cytoplasmic retention of CIC in Drosophila melanogaster and mammals [20, 32, 48], the decreased expression of CIC in samples obtained from CRC patients may result from enhanced activity of MAPKs. Reduced expression of CIC proteins was also observed in tissue samples from patients with other types of cancers, such as prostate and liver cancers [36, 37]. Therefore, a decrease in CIC levels may be one of the key features occurring in the process of cancer progression in various types of cancers that exhibit hyperactivation of RAS/MAPK signaling.

Alterations in several essential developmental signaling pathways including WNT, NOTCH, and Sonic hedgehog $(\mathrm{SHH})$ are known to be associated with CRC progression [49] Among these, oncogenic mutations in 


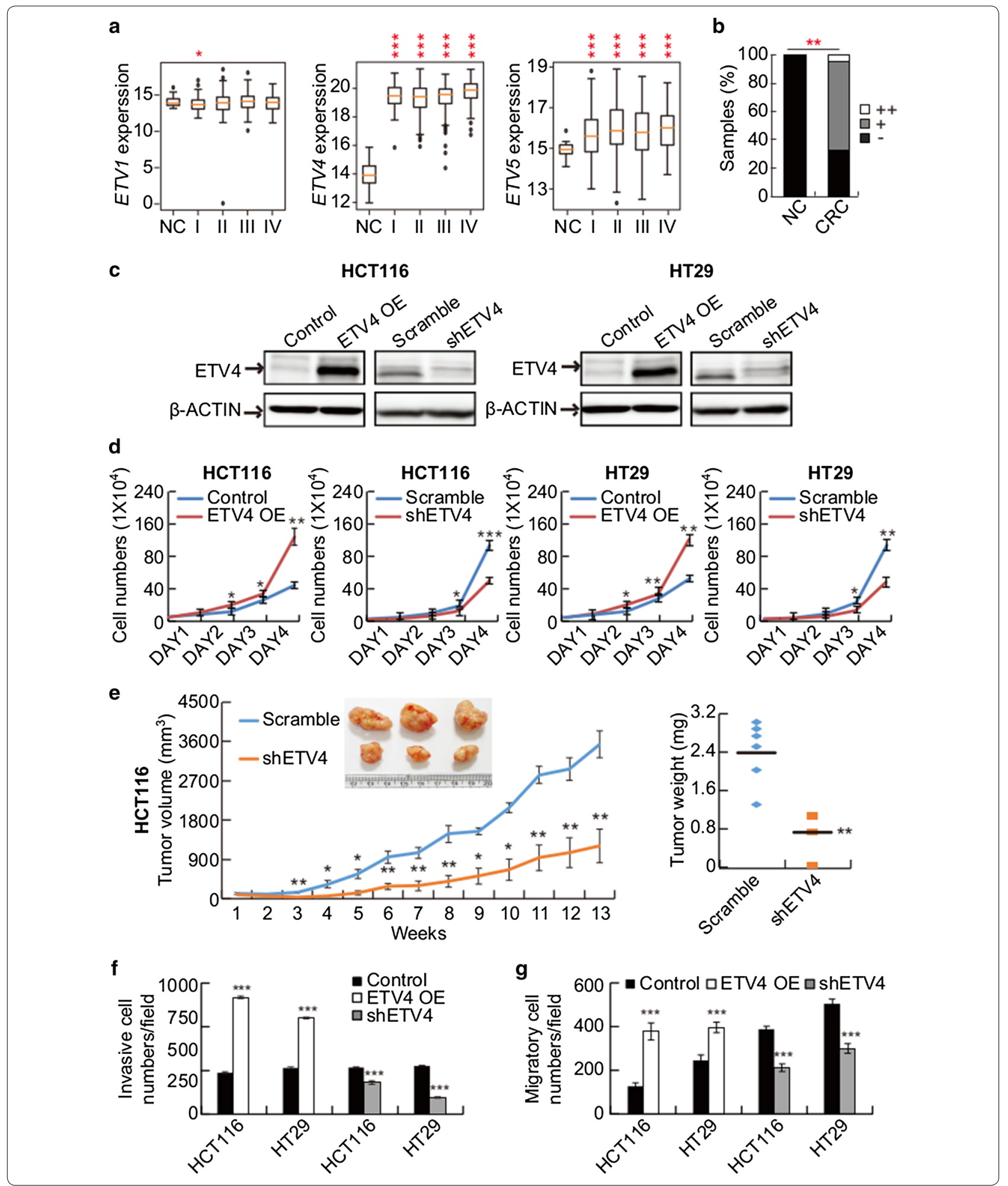

the WNT pathway genes are prevalent in CRC. Mutations inactivating $A P C$ are found in $70-80 \%$ of CRCs, and these are believed to initiate malignant transformation of the colorectal epithelial cells $[7,49]$. However, the majority of APC mutant colon epithelial tumors are benign and never progress to $C R C$, suggesting that other genetic 
a

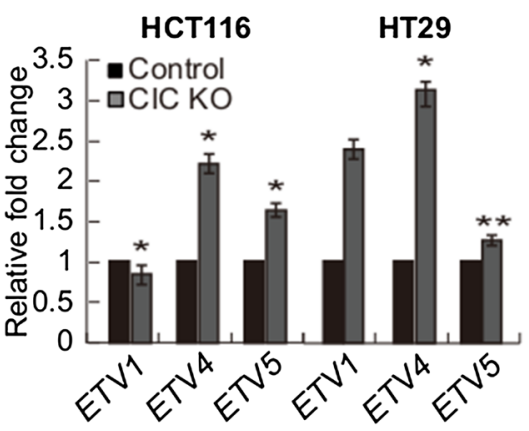

C
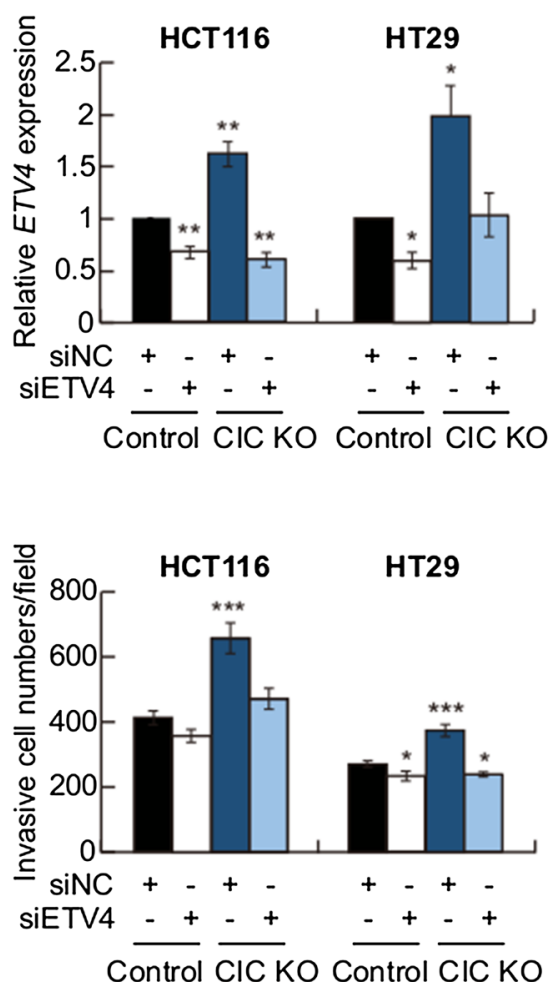

b

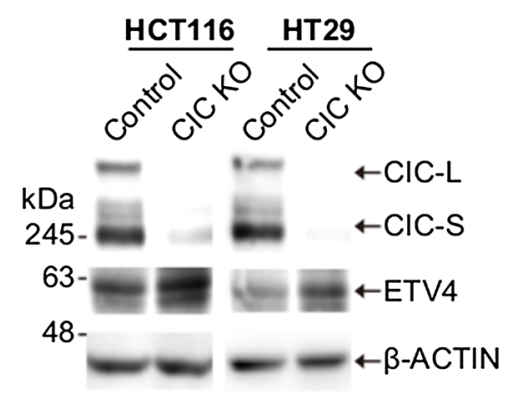

d

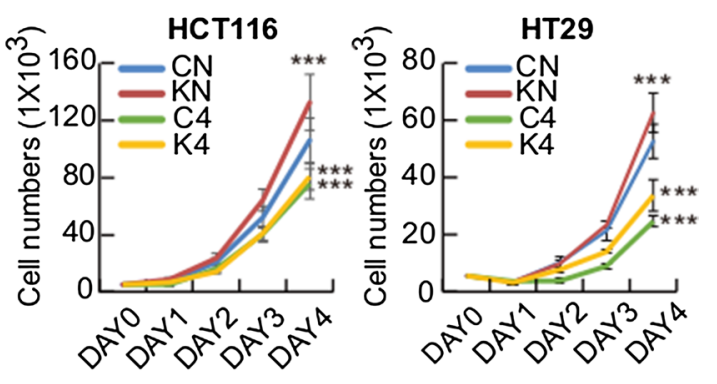

f

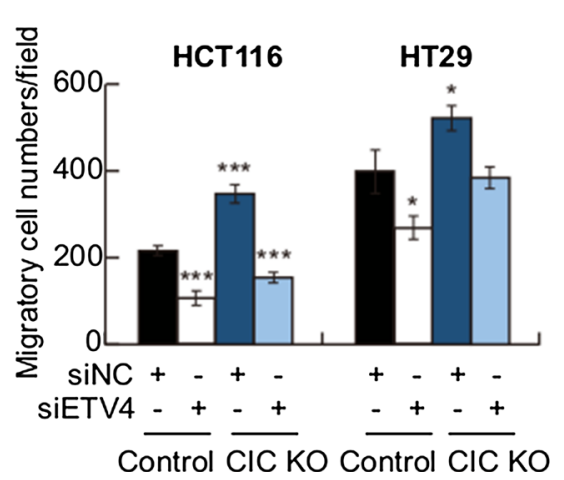

Fig. $4 \mathrm{CIC}$ deficiency-mediated promotion of CRC progression is due to derepression of ETV4. a qRT-PCR analysis of PEA3 group gene expression levels in control and CIC-deficient (CIC KO) CRC cells (HCT116 and HT29). b Western blot analysis presenting upregulation of ETV4 expression in CIC KO CRC cells. c qRT-PCR analysis for ETV4 mRNA levels in control and CIC KO CRC cells transfected with either control (siNC) or ETV4 siRNA (siETV4). $\mathbf{d}-\mathbf{f}$ Cell growth assay (d), Matrigel invasion assay (e), and trans-well migration assay $(\mathbf{f})$ of control and CIC knockout CRC cells treated with either siNC or siETV4. Three independent experiments were performed. All error bars indicate s.e.m. ${ }^{*} P<0.05$, ${ }^{* *} P<0.01$, and ${ }^{* * *} P<0.001$

alterations are required for the development of WNT signaling-mutant colon epithelia into CRC. Given this, it is conceivable that CIC might be a gene whose loss or inactivating mutations drive CRC development and progression via collaboration with the WNT pathway. Consistent with this, ETV4 stabilizes $\beta$-catenin, a key transcription factor mediating WNT signaling, to promote tumor aggressiveness in gastrointestinal stromal tumors [50]. In future studies, it will be interesting to explore if and how the CIC-ETV4 axis cross-talks with the major signaling pathways such as WNT signaling that are altered in CRC cells.

\section{Conclusions}

This is the first study to demonstrate that $\mathrm{CIC}$ functions as a tumor suppressor in CRC cells. Our findings also highlight ETV4 as a strong promoter of cancer progression as well as a critical target of CIC in the context of CRC. In conclusion, the CIC-ETV4 axis is a key molecular module that controls CRC progression. 


\section{Supplementary information}

Supplementary information accompanies this paper at https://doi. org/10.1186/s12935-020-1111-8.

Additional file 1: Table S1. List of CIC mutations in various types of cancers from the International Cancer Genome Consortium (ICGC) database. Table S2. Clinical and pathological characteristics of the COAD patients in TCGA. Table S3. List of 9 CRC patient samples (for Fig. 1C, Additional file 3: Fig. S2 and Additional file 4: Fig. S3). CRC patient samples were provided by Soonchunhyang University Hospital (South Korea). Table S4. List of 4 CRC patients (for Fig. 1d). CRC patient samples were provided by Soonchunhyang University Hospital (South Korea).

Additional file 2: Figure S1. Mutations and expression levels of $C / C$ in CRC patient samples. a Schematic illustration of the location of point mutations in C/C identified from patients with colorectal cancer. The image was captured from the CBioPortal database. b Analyses of two different datasets (GSE5206 and GSE20916) from COSMIC database for CIC mRNA levels in normal and colorectal tumor tissues. The numbers in parentheses indicate the number of subjects in each group.

Additional file 3: Figure S2. Reduced expression of CIC in colorectal tumors. Immunohistochemical staining of tissue samples from patients with CRC using anti-CIC antibody. CIC expression is substantially decreased in the tumor areas compared with that in the normal colon areas.

Additional file 4: Figure S3. Increased expression ETV4 in colorectal tumors. Immunohistochemical staining of tissue samples from patients with CRC using anti-ETV4 antibody. ETV4 expression is dramatically increased in the tumor areas compared with that in the normal colon areas.

\section{Abbreviations}

CRC: colorectal cancer; CIC: Capicua; PEA3: polyoma enhancer activator 3; ETV4: ETS translocation variant 4; EGFR: epidermal growth factor receptor; HMG: high mobility group; TCGA: The Cancer Genome Atlas; COSMIC: Catalogue of Somatic Mutations in Cancer; CIC KO: CIC-deficient; MAPK: mitogen activated protein kinase.

\section{Acknowledgements}

We thank Lee lab members and Dr. Jooyeon Yoo (POSTECH, Korea) for their helpful discussion on this study and provision of CRC cell lines, respectively.

\section{Authors' contributions}

J-SL, EK, and YL designed the study. DJ provided a clinical insight on the relevance of CIC and ETV4 for the pathogenesis of CRC. J-SL, EK, and JL performed a majority of experiments in this study. DK and SK conducted bioinformatic analyses of gene expression profile datasets for the CRC patients. HK, C-JK, and DJ provided human CRC tissue samples. C-JK and DJ evaluated the ETV4 expression levels in the CRC tissue samples of tissue microarrays. EK, JL, and $Y L$ wrote the manuscript. All authors read and approved the final manuscript.

\section{Funding}

This work was supported by Grants from the National Research Foundation of Korea (NRF) funded by the Korean Ministry of Science and ICT (2018R1A2B2004416 and 2017R1A5A1015366), the BK21 Plus Program (Program of Bio-Molecular Function, POSTECH), the POSCO green science program, and TJ Park Science Fellowship of POSCO TJ Park Foundation. JL was supported by Global PhD Fellowship (NRF-2018H1A2A1059794).

\section{Availability of data and materials}

All data generated or analyzed during this study are available from the corresponding author on reasonable request.

\section{Ethics approval and consent to participate}

The experiments with the human specimens were approved by the Soonchunhyang University Hospital Institutional Review Board (SCHCA 2018-07-061-003). All animal studies were performed in compliance with the Pohang University of Science and Technology Institutional Animal Care and
Use Committee (IACUC) guidelines. The study protocols were approved by POSTECH IACUC (POSTECH-2018-0063) and complied with the regulations by Animal and Plant Quarantine Agency of Republic of Korea.

\section{Consent for publication}

Not applicable yet.

\section{Competing interests}

The authors declare that they have no competing interests.

\section{Author details}

1 Department of Life Sciences, Pohang University of Science and Technology, Pohang, Gyeongbuk, Republic of Korea. ${ }^{2}$ Division of Integrative Bioscience and Biotechnology, Pohang University of Science and Technology, Pohang, Gyeongbuk, Republic of Korea. ${ }^{3}$ Soonchunhyang Medical Science Research Institute, Soonchunhyang University, Cheonan, Chungnam, Republic of Korea. ${ }^{4}$ Department of Pathology, College of Medicine, Soonchunhyang University, Room 601, 31 Soonchunhyang 6gil, Dongnam-gu, Cheonan, Chungnam 31151, Republic of Korea. ${ }^{5}$ POSTECH Biotech Center, Room 388, 77 Cheongam-Ro, Nam-Gu, Pohang, Gyeongbuk 37673, Republic of Korea.

Received: 29 April 2019 Accepted: 17 January 2020

Published online: 05 February 2020

\section{References}

1. Bray F, Ferlay J, Soerjomataram I, Siegel RL, Torre LA, Jemal A. Global cancer statistics 2018: GLOBOCAN estimates of incidence and mortality worldwide for 36 cancers in 185 countries. CA Cancer J Clin. 2018:68(6):394-424.

2. Baker SJ, Preisinger AC, Jessup JM, Paraskeva C, Markowitz S, Willson J, et al. p53 gene mutations occur in combination with 17p allelic deletions as late events in colorectal tumorigenesis. Cancer Res. 1990:50(23):7717-22

3. Kinzler KW, Vogelstein B. Lessons from hereditary colorectal cancer. Cell. 1996;87(2):159-70.

4. Chan TL, Zhao W, Leung SY, Yuen ST. BRAF and KRAS mutations in colorectal hyperplastic polyps and serrated adenomas. Cancer Res. 2003:63(16):4878-81.

5. Malumbres M, Barbacid M. RAS oncogenes: the first 30 years. Nat Rev Cancer. 2003:3(6):459.

6. Pretlow TP, Pretlow TG. Mutant KRAS in aberrant crypt foci (ACF): initiation of colorectal cancer? Biochim Biophys Acta. 2005;1756(2):83-96.

7. Segditsas S, Tomlinson I. Colorectal cancer and genetic alterations in the Wnt pathway. Oncogene. 2006:25(57):7531.

8. Meyerhardt JA, Mayer RJ. Systemic therapy for colorectal cancer. N Engl J Med. 2005;352(5):476-87.

9. Kawamura-Saito M, Yamazaki Y, Kaneko K, Kawaguchi N, Kanda H, Mukai $\mathrm{H}$, et al. Fusion between CIC and DUX4 up-regulates PEA3 family genes in Ewing-like sarcomas with t $(4 ; 19)(q 35 ;$ q13) translocation. Hum Mol Genet. 2006;15(13):2125-37.

10. Löhr U, Chung H-R, Beller M, Jäckle H. Antagonistic action of Bicoid and the repressor Capicua determines the spatial limits of Drosophila head gene expression domains. Proc Natl Acad Sci. 2009;106(51):21695-700.

11. Ajuria L, Nieva C, Winkler C, Kuo D, Samper N, Andreu MJ, et al. Capicua DNA-binding sites are general response elements for RTK signaling in Drosophila. Development. 2011;138(5):915-24.

12. Jiménez G, Shvartsman SY, Paroush ZE. The Capicua repressor-a general sensor of RTK signaling in development and disease. J Cell Sci. 2012;125(6):1383-91.

13. Krivy K, Bradley-Gill M-R, Moon N-S. Capicua regulates proliferation and survival of RB-deficient cells in Drosophila. Biol Open. 2013;2(2):183-90.

14. Fores M, Simon-Carrasco L, Ajuria L, Samper N, Gonzalez-Crespo S, Drosten $M$, et al. A new mode of DNA binding distinguishes Capicua from other HMG-box factors and explains its mutation patterns in cancer. PLoS Genet. 2017;13(3):e1006622.

15. Shin D-H, Hong J-W. Capicua is involved in Dorsal-mediated repression of zerknüllt expression in Drosophila embryo. BMB Rep. 2014;47(9):518. 
16. Weissmann S, Cloos PA, Sidoli S, Jensen ON, Pollard S, Helin K. The tumor suppressor $\mathrm{CIC}$ directly regulates MAPK pathway genes via histone deacetylation. Cancer Res. 2018;78(15):4114-25.

17. Lam YC, Bowman AB, Jafar-Nejad P, Lim J, Richman R, Fryer JD, et al. ATAXIN-1 interacts with the repressor Capicua in its native complex to cause SCA1 neuropathology. Cell. 2006;127(7):1335-47.

18. Chittaranjan S, Chan S, Yang C, Yang KC, Chen V, Moradian A, et al. Mutations in $\mathrm{CIC}$ and IDH1 cooperatively regulate 2-hydroxyglutarate levels and cell clonogenicity. Oncotarget. 2014;5(17):7960.

19. Astigarraga S, Grossman R, Díaz-Delfín J, Caelles C, Paroush ZE, Jimenez G. A MAPK docking site is critical for downregulation of Capicua by Torso and EGFR RTK signaling. EMBO J. 2007;26(3):668-77.

20. Grimm O, Zini VS, Kim Y, Casanova J, Shvartsman SY, Wieschaus E. Torso RTK controls Capicua degradation by changing its subcellular localization. Development. 2012;139(21):3962-8.

21. Lim B, Samper N, Lu H, Rushlow C, Jiménez G, Shvartsman SY. Kinetics of gene derepression by ERK signaling. Proc Natl Acad Sci. 2013;110(25):10330-5.

22. Jin Y, Ha N, Forés M, Xiang J, Gläßer C, Maldera J, et al. EGFR/Ras signaling controls Drosophila intestinal stem cell proliferation via Capicua-regulated genes. PLoS Genet. 2015;11(12):e1005634.

23. Yang L, Paul S, Trieu KG, Dent LG, Froldi F, Forés M, et al. Minibrain and Wings apart control organ growth and tissue patterning through downregulation of Capicua. Proc Natl Acad Sci. 2016;1 13(38):10583-8.

24. Lee Y, Fryer JD, Kang H, Crespo-Barreto J, Bowman AB, Gao Y, et al. ATXN1 protein family and $\mathrm{CIC}$ regulate extracellular matrix remodeling and lung alveolarization. Dev Cell. 2011;21(4):746-57.

25. Kim E, Park S, Choi N, Lee J, Yoe J, Kim S, et al. Deficiency of Capicua disrupts bile acid homeostasis. Sci Rep. 2015;5:8272.

26. Lu H-C, Tan Q, Rousseaux MW, Wang W, Kim J-Y, Richman R, et al. Disruption of the ATXN1-CIC complex causes a spectrum of neurobehavioral phenotypes in mice and humans. Nat Genet. 2017;49(4):527.

27. Park S, Lee S, Lee C-G, Park GY, Hong H, Lee J-S, et al. Capicua deficiency induces autoimmunity and promotes follicular helper T cell differentiation via derepression of ETV5. Nat Commun. 2017:8:16037.

28. Park S, Park J, Kim E, Lee Y. The Capicua/ETS translocation variant 5 axis regulates liver-resident memory CD8+T-cell development and the pathogenesis of liver injury. Hepatology. 2019;70(1):358-371.

29. Sjöblom T, Jones S, Wood LD, Parsons DW, Lin J, Barber TD, et al. The consensus coding sequences of human breast and colorectal cancers. Science. 2006;314(5797):268-74.

30. Kan Z, Jaiswal BS, Stinson J, Janakiraman V, Bhatt D, Stern HM, et al. Diverse somatic mutation patterns and pathway alterations in human cancers. Nature. 2010;466(7308):869.

31. Bettegowda C, Agrawal N, Jiao Y, Sausen M, Wood LD, Hruban RH, et al. Mutations in CIC and FUBP1 contribute to human oligodendroglioma. Science. 2011;333(6048):1453-5.

32. Okimoto RA, Breitenbuecher F, Olivas VR, Wu W, Gini B, Hofree M, et al. Inactivation of Capicua drives cancer metastasis. Nat Genet. 2017:49(1):87.

33. Graham C, Chilton-MacNeill S, Zielenska M, Somers GR. The CIC-DUX4 fusion transcript is present in a subgroup of pediatric primitive round cell sarcomas. Hum Pathol. 2012:43(2):180-9.

34. Italiano A, Sung YS, Zhang L, Singer S, Maki RG, Coindre JM, et al. High prevalence of CIC fusion with double-homeobox (DUX4) transcription factors in EWSR1-negative undifferentiated small blue round cell sarcomas. Genes Chromosom Cancer. 2012;51(3):207-18.
35. Yoshida A, Goto K, Kodaira M, Kobayashi E, Kawamoto H, Mori T, et al. CICrearranged sarcomas. Am J Surg Pathol. 2016;40(3):313-23.

36. Choi N, Park J, Lee J-S, Yoe J, Park GY, Kim E, et al. miR-93/miR-106b/miR375-CIC-CRABP1: a novel regulatory axis in prostate cancer progression. Oncotarget. 2015;6(27):23533.

37. Kim E, Kim D, Lee JS, Yoe J, Park J, Kim CJ, et al. Capicua suppresses hepatocellular carcinoma progression by controlling the ETV4-MMP1 axis. Hepatology. 2018;67(6):2287-301.

38. Oh S, Shin S, Janknecht R. ETV1, 4 and 5: an oncogenic subfamily of ETS transcription factors. Biochim Biophys Acta. 2012;1826(1):1-12.

39. Seshagiri S, Stawiski EW, Durinck S, Modrusan Z, Storm EE, Conboy $\mathrm{CB}$, et al. Recurrent R-spondin fusions in colon cancer. Nature. 2012;488(7413):660

40. Simón-Carrasco L, Jiménez G, Barbacid M, Drosten M. The Capicua tumor suppressor: a gatekeeper of Ras signaling in development and cancer. Cell Cycle. 2018;17(6):702-11.

41. Dissanayake K, Toth R, Blakey J, Olsson O, Campbell DG, Prescott AR, et al. ERK/p90RSK/14-3-3 signalling has an impact on expression of PEA3 Ets transcription factors via the transcriptional repressor capicua. Biochem J. 2011:433(3):515-25

42. Fryer JD, Yu P, Kang H, Mandel-Brehm C, Carter AN, Crespo-Barreto J, et al. Exercise and genetic rescue of SCA1 via the transcriptional repressor Capicua. Science. 2011;334(6056):690-3.

43. Sizemore GM, Pitarresi JR, Balakrishnan S, Ostrowski MC. The ETS family of oncogenic transcription factors in solid tumours. Nat Rev Cancer. 2017;17(6):337

44. Moss AC, Lawlor G, Murray D, Tighe D, Madden SF, Mulligan A-M, et al. ETV4 and Myeov knockdown impairs colon cancer cell line proliferation and invasion. Biochem Biophys Res Commun. 2006;345(1):216-21.

45. Mesci A, Taeb S, Huang X, Jairath R, Sivaloganathan D, Liu SK. Pea3 expression promotes the invasive and metastatic potential of colorectal carcinoma. World J Gastroenterol. 2014;20(46):17376.

46. Rajagopalan H, Bardelli A, Lengauer C, Kinzler KW, Vogelstein B, Velculescu VE. Tumorigenesis: RAF/RAS oncogenes and mismatch-repair status. Nature. 2002:418(6901):934.

47. Heinemann V, Stintzing S, Kirchner T, Boeck S, Jung A. Clinical relevance of EGFR-and KRAS-status in colorectal cancer patients treated with monoclonal antibodies directed against the EGFR. Cancer Treat Rev. 2009;35(3):262-71.

48. Bunda S, Heir P, Metcalf J, Li ASC, Agnihotri S, Pusch S, et al. CIC protein instability contributes to tumorigenesis in glioblastoma. Nat Commun. 2019;10(1):661.

49. Faroogi AA, De La Roche M, Djamgoz MB, Siddik ZH, editors. Overview of the oncogenic signaling pathways in colorectal cancer: Mechanistic insights. In: Seminars in cancer biology. Elsevier; 2019.

50. Zeng S, Seifert AM, Zhang JQ, Kim TS, Bowler TG, Cavnar MJ, et al. ETV4 collaborates with Wnt/ $\beta$-catenin signaling to alter cell cycle activity and promote tumor aggressiveness in gastrointestinal stromal tumor. Oncotarget. 2017;8(69):114195

\section{Publisher's Note}

Springer Nature remains neutral with regard to jurisdictional claims in published maps and institutional affiliations.

Ready to submit your research? Choose BMC and benefit from:

- fast, convenient online submission

- thorough peer review by experienced researchers in your field

- rapid publication on acceptance

- support for research data, including large and complex data types

- gold Open Access which fosters wider collaboration and increased citations

- maximum visibility for your research: over 100M website views per year

At BMC, research is always in progress.

Learn more biomedcentral.com/submissions 